\title{
Pulse-train control of photofragmentation at constant field energy
}

Tiwari, Ashwani Kumar; Henriksen, Niels Engholm

Published in:

Journal of Chemical Physics

Link to article, DOI:

$10.1063 / 1.4902061$

Publication date:

2014

Document Version

Publisher's PDF, also known as Version of record

Link back to DTU Orbit

Citation (APA):

Tiwari, A. K., \& Henriksen, N. E. (2014). Pulse-train control of photofragmentation at constant field energy. Journal of Chemical Physics, 141(20), 204301. [204301]. https://doi.org/10.1063/1.4902061

\section{General rights}

Copyright and moral rights for the publications made accessible in the public portal are retained by the authors and/or other copyright owners and it is a condition of accessing publications that users recognise and abide by the legal requirements associated with these rights.

- Users may download and print one copy of any publication from the public portal for the purpose of private study or research.

- You may not further distribute the material or use it for any profit-making activity or commercial gain

- You may freely distribute the URL identifying the publication in the public portal

If you believe that this document breaches copyright please contact us providing details, and we will remove access to the work immediately and investigate your claim 


\section{AD| $\begin{aligned} & \text { The Journal of } \\ & \text { Chemical Physics }\end{aligned}$}

\section{Pulse-train control of photofragmentation at constant field energy}

Ashwani K. Tiwari and Niels E. Henriksen

Citation: The Journal of Chemical Physics 141, 204301 (2014); doi: 10.1063/1.4902061

View online: http://dx.doi.org/10.1063/1.4902061

View Table of Contents: http://scitation.aip.org/content/aip/journal/jcp/141/20?ver=pdfcov

Published by the AIP Publishing

\section{Articles you may be interested in}

Strong-field induced XUV transmission and multiplet splitting in 4d-16p core-excited Xe studied by femtosecond XUV transient absorption spectroscopy

J. Chem. Phys. 137, 244305 (2012); 10.1063/1.4772199

Control of resonance enhanced multi-photon ionization photoelectron spectroscopy by phase-shaped femtosecond laser pulse

J. Chem. Phys. 137, 174301 (2012); 10.1063/1.4762865

Pulse-train control of branching processes: Elimination of background and intruder state population

J. Chem. Phys. 129, 234305 (2008); 10.1063/1.3041380

Energy distribution of ionized electrons from He atoms in strong laser fields

Phys. Plasmas 7, 414 (2000); 10.1063/1.873810

Real time study of bimolecular interactions: Direct detection of internal conversion involving $\operatorname{Br}(2 \mathrm{P} 1 / 2)+12$ initiated from a van der Waals dimer

J. Chem. Phys. 106, 2634 (1997); 10.1063/1.473366

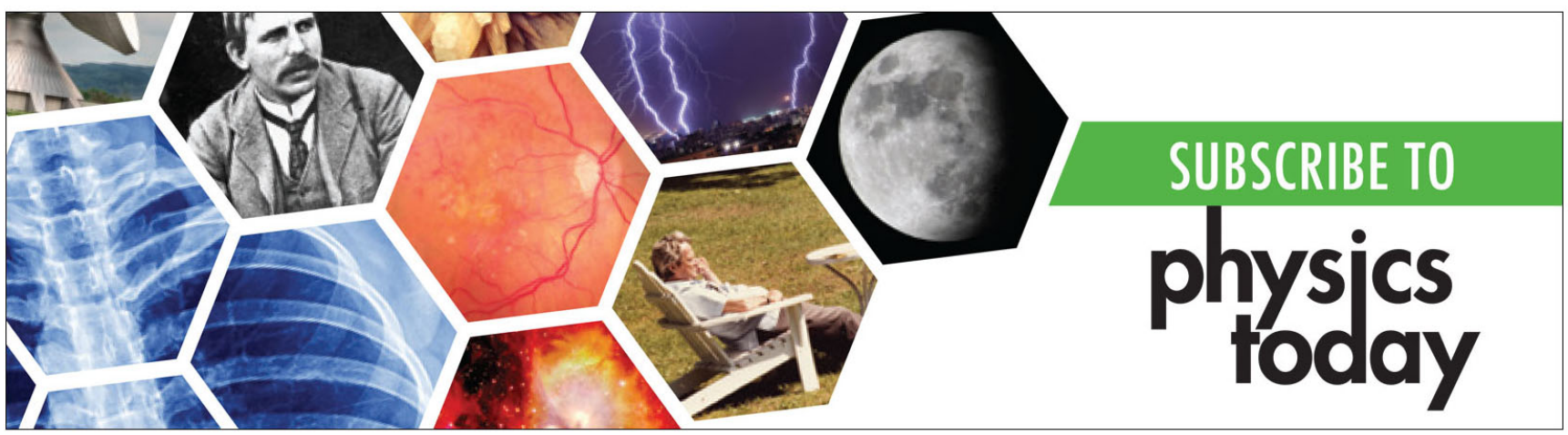




\title{
Pulse-train control of photofragmentation at constant field energy
}

\author{
Ashwani K. Tiwari ${ }^{1, a)}$ and Niels E. Henriksen ${ }^{2, b)}$ \\ ${ }^{1}$ Indian Institute of Science Education and Research Kolkata, Mohanpur 741 252, India \\ ${ }^{2}$ Department of Chemistry, Building 207, Technical University of Denmark, DK-2800 Kgs. Lyngby, Denmark
}

(Received 8 September 2014; accepted 6 November 2014; published online 24 November 2014)

\begin{abstract}
We consider a phaselocked two-pulse sequence applied to photofragmentation in the weak-field limit. The two pulses are not overlapping in time, i.e., the energy of the pulse-train is constant for all time delays. It is shown that the relative yield of excited $\mathrm{Br}^{*}$ in the nonadiabatic process: $\mathrm{I}+\mathrm{Br}^{*} \leftarrow \mathrm{IBr} \rightarrow \mathrm{I}+\mathrm{Br}$, changes as a function of time delay when the two excited wave packets interfere. The underlying mechanisms are analyzed and the change in the branching ratio as a function of time delay is only a reflection of a changing frequency distribution of the pulse train; the branching ratio does not depend on the detailed pulse shape. (C) 2014 AIP Publishing LLC. [http://dx.doi.org/10.1063/1.4902061]
\end{abstract}

\section{INTRODUCTION}

The use of optimized laser fields to guide the dynamics of an atom or molecule from a given initial state into a desired final state is a topic of much current interest. ${ }^{1-9}$ To be specific, we consider in the following a molecule which is bound in its electronic ground state and where fragmentation can take place in excited electronic states. In the weak-field limit, shaping of the laser field can modify the dynamics. In the strong-field limit, the laser fields can modify the dynamics as well as potential energy surfaces. Selective shaping of potential energy surfaces has recently been demonstrated. ${ }^{10,11}$

The time-dependent phase-coherent electric field of a laser pulse can be represented by

$$
\mathcal{E}(t)=\mathcal{E}_{0} \operatorname{Re}\left[\int_{-\infty}^{\infty} A(\omega) e^{i \phi(\omega)} e^{-i \omega t} d \omega\right],
$$

where $A(\omega)$ is the real-valued distribution of frequencies and $\phi(\omega)$ is the real-valued frequency-dependent phase. The energy in the field $\propto \int|\mathcal{E}(t)|^{2} d t \propto \int\left|A(\omega) e^{i \phi(\omega)}\right|^{2} d \omega$ $=\int|A(\omega)|^{2} d \omega$ is completely determined by the frequency distribution and is independent of the phases. Genuine/active coherent laser control is defined as control which depends on the phases $\phi(\omega)$. These phases modify the pulse shape and can affect quantum interferences.

In the weak-field (one-photon) limit, amplitude is exclusively transferred from the electronic ground state to excited state surfaces. However, when excitation out of a single eigenstate of a molecule is considered and direct fragmentation takes place within a dissociative continuum of states, a seminal proof showed that no phase control of final state distributions of the fragments is possible in the long-time limit. ${ }^{12,13}$ Thus, the total excitation probability into a particular arrangement channel of an excited (ex) electronic state with nuclear Hamiltonian $H_{\mathrm{ex}}$ is ${ }^{14}$

$$
P_{\mathrm{ex}}=\int_{\text {all } E \text { in ex. }}|A(E / \hbar)|^{2} P(E) d E
$$

\footnotetext{
a)Electronic mail: ashwani@iiserkol.ac.in

b)Electronic mail: neh@kemi.dtu.dk
}

with $P(E)=\mathcal{C} \sum_{n} \lim _{t \rightarrow \infty}\left|\left\langle E, n\left|\exp \left(-i H_{\mathrm{ex}} t / \hbar\right)\right| \phi(0)\right\rangle\right|^{2}$, $|\phi(0)\rangle$ is the initial eigenstate (e.g., the vibrational ground state) times the projection of the transition dipole moment along the polarization direction of the field, $|E, n\rangle$ is an eigenstate of the chosen arrangement channel of a product with degenerate states labeled by $n$, and the integration is over all energies $E$ in the excited electronic state (measured relative to the energy of the initial state).

In this limit, according to Eq. (2), only the frequency distribution of the laser field is reflected in the excitation probability, i.e., via $|A(\omega)|^{2}$, where $\omega=E / \hbar$. A short laser pulse generates products with a distribution of energies selected according to $|A(\omega)|^{2}$. The outcome which is obtained with some particular pulse shape of an ultrashort pulse can, in principle, always be reproduced by a set of (incoherent) dissociation processes induced by cw-pulses where $|A(\omega)|^{2}=\delta(\omega$ $\left.-\omega_{0}\right)$ and $P_{\mathrm{ex}}=P\left(E_{0}=\hbar \omega_{0}\right)$. Thus, the set of cw-pulses must have different intensities and frequencies corresponding to the frequency distribution $|A(\omega)|^{2}$ of the short pulse.

Laser phase dependence can, however, be observed for weak-field excitation out of a nonstationary superposition of bound vibrational states. ${ }^{2,15-19}$ Furthermore, beyond the weak-field limit, coherent control plays an important role in the pulsed strong-field excitation out of a single stationary vibrational state of a molecule. In the strong-field limit, amplitude can be transferred from the electronic ground state to an excited electronic state and back to the electronic ground state. This can lead to a nonstationary superposition of bound vibrational states which subsequently is transferred to an excited electronic state where fragmentation takes place. ${ }^{20,21}$ The use of strong fields create, however, potential problems with the population of unwanted channels, e.g., related to ionization. $^{22}$

Recently, the weak-field excitation out of a single stationary state has again attracted attention. ${ }^{23-30}$ Thus, phase dependence of isomerization yields has been reported recently in the weak-field limit. ${ }^{23-26}$ It was shown that phase dependence can persist over long times when the few modes that are active in the isomerization are coupled intramolecularly to the 


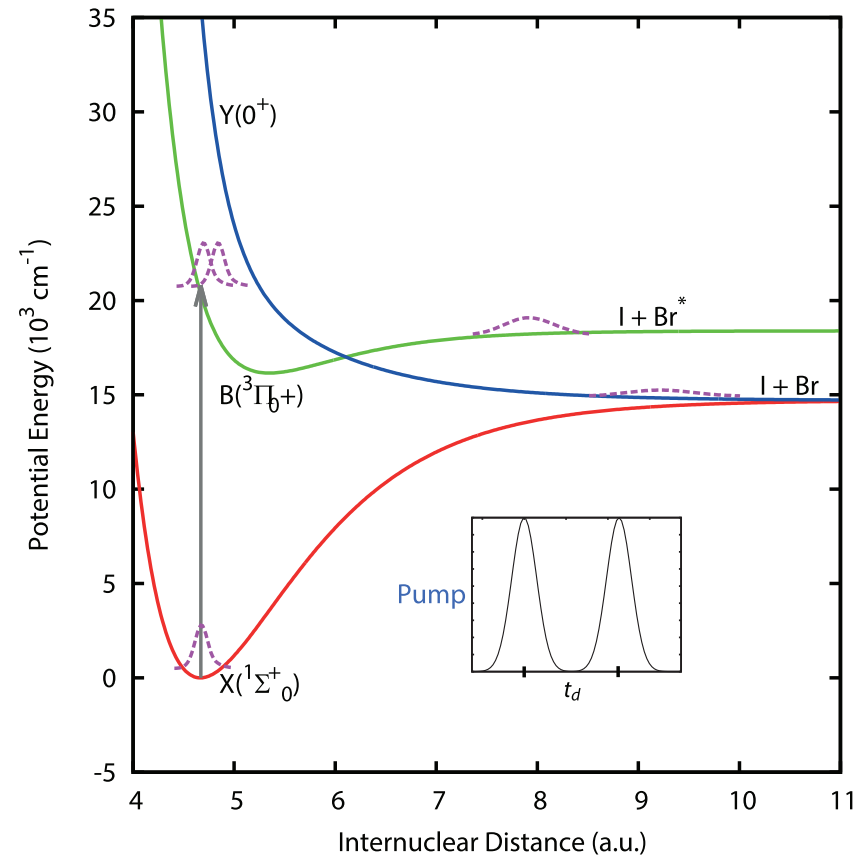

FIG. 1. The relevant diabatic potential energy curves of IBr with the twopulse pump sequence, at time delay $t_{d}$, illustrated in the inset. The excited interfering wave packets are sketched right after the pump has decayed. Due to the nonadiabatic crossing, the wave packets bifurcate into the two channels $\mathrm{I}+\mathrm{Br}^{*}$ and $\mathrm{I}+\mathrm{Br}$.

many vibrational modes in a large molecule or, in general, to an external environment. This coupling allows for dissipation of energy from the isomerization coordinates and effectively constrains the observables to finite times of the system dynamics. It has even been predicted that cis/trans branching ratios can be phase controlled without a requirement for an open system. ${ }^{27}$

As mentioned above, for direct fragmentation in the longtime limit, it has been shown that genuine/active laser control is not possible. ${ }^{12}$ However, we showed recently that a phase dependence associated with a fixed bandwidth phase modulated pulse can be observed and persists for some time after the pulse is over-also for direct fragmentation within an isolated dissociative continuum with a few degrees of freedom. Not only the total dissociation probability ${ }^{28,29}$ but also the branching ratio between two channels can be modified within a certain time interval. ${ }^{30}$

In this paper we explore again laser control in the excitation out of a single eigenstate of a molecule in the weak-field limit. We consider specifically the process

$$
\mathrm{IBr}+\text { coherent light } \rightarrow\left\{\begin{array}{l}
\mathrm{I}+\mathrm{Br}^{*} \\
\mathrm{I}+\mathrm{Br}
\end{array},\right.
$$

where $\mathrm{Br}^{*}$ is the spin-orbit excited $\operatorname{Br}\left({ }^{2} \mathrm{P}_{1 / 2}\right)$. IBr is initially in its vibrational ground state. The relevant potential energy curves are shown in Fig. 1. The IBr branching ratio has previously been the subject of coherent laser control studies. ${ }^{30,31}$

It is well known ${ }^{32}$ that the laser excitation process can be thought of as consisting of a coherent excitation of FranckCondon wave packets, $\exp \left(-i H_{\mathrm{ex}} t^{\prime} / \hbar\right)|\phi(0)\rangle$, each of which corresponds to a delta-pulse excitation at time $t^{\prime}$. The result- ing excited-state wave function at time $t$ is a coherent superposition of these wave packets, time-evolved for a time $t-t^{\prime}$.

Intriguing demonstrations of this picture, involving quantum interference of nuclear wave packets in an excited electronic state of a molecule, have been published. For example, the observation of constructive or destructive interference giving rise to larger or smaller excited state population ${ }^{33,34}$ and the direct observation, via Coulomb explosion, of the nuclear probability density resulting from quantum interference of two wave packets. ${ }^{35}$ The implications of wave packet interference for the total excitation probability have also been nicely discussed from a theoretical point of view. ${ }^{36}$

In the context of control, we consider here the implications of such wave packet interference for the relative yield of the two channels in Eq. (3). Using pulse-train excitation, with ultrashort non-overlapping pulses, we excite here with a fixed pulse energy as a function of time delay between the pulses. Pulse trains have previously been applied in order to control the population of excited molecular states see, e.g., Refs. 33, 34, 37, and 38.

The rest of this paper is organized as follows: In Sec. II., the molecular system and the theoretical and computational approaches are briefly presented. In Sec. III., the results are presented and discussed. Finally, conclusions are given in Sec. IV.

\section{THEORETICAL/COMPUTATIONAL APPROACH}

A detailed discussion of the electronic states of $\mathrm{IBr}$ can be found in Ref. 39 (and references therein). For our purpose, the simple 3-state model shown in Fig. 1 is sufficient. ${ }^{10,40,41}$ The two excited state potentials interact with each other around their crossing at an internuclear distance of about 6 a.u. We consider in the following laser excitation out of the vibrational and electronic ground state where vertical excitation corresponds to a wavelength of about $\lambda=500 \mathrm{~nm}$. Essentially direct dissociation takes place for $\lambda<545 \mathrm{~nm}$, which is above the dissociation limit for both channels. This three-state model $^{41}$ reproduces experimental observations at low energies (i.e., $\lambda>500 \mathrm{~nm}$ ) additional excited electronic states are involved at higher energies. ${ }^{40}$ The laser field consists of two ultrashort Gaussian pulses with a variable time delay and a fixed phase relationship. Thus,

$$
\mathcal{E}(t)=E_{1}(t)+E_{2}(t)
$$

where

$$
\begin{aligned}
& E_{1}(t)=E_{0} \exp \left[-t^{2} / 2 \tau^{2}\right] \cos (\omega t), \\
& E_{2}(t)=E_{0} \exp \left[-\left(t-t_{d}\right)^{2} / 2 \tau^{2}\right] \cos \left[\omega\left(t-t_{d}\right)+\phi_{r}\right] .
\end{aligned}
$$

Here $\omega$ is the center frequency (equivalent to the wavelength $\lambda_{0}=500 \mathrm{~nm}$ ) of each pulse and $\phi_{r}$ is the relative phase of the two subpulses. The duration of each pulse is in the following set to $2 \mathrm{fs}$. We choose the time delay, $t_{d}$, such that the two subpulses are not overlapping, i.e., the energy of the field is constant and equal to two times the energy in each subpulse.

We treat IBr as a one-dimensional system (see Ref. 41 for details), assuming that the transition dipole moment for the $X \rightarrow B$ transition is oriented in the direction of the field. 
In this description, the angular momentum of the overall rotational motion, i.e., the centrifugal energy is neglected in the dynamics of the internuclear motion. This is an excellent approximation, at least, for small values of the angular momentum. ${ }^{28}$ The laser-induced dynamics is calculated within the electric-dipole approximation and first-order perturbation theory for the interaction with the field.

The wave functions, potentials and coupling element are represented on an equally spaced grid of 1024 points with $3.70 \leq x \leq 20.50$ a.u. An absorbing potential is added for $x \geq 18.4$ a.u. to avoid unphysical reflections into the inner region. In the diabatic representation, the time propagation of wave functions, $\psi_{i}(x, t)$ where $i$ refers to channel $\mathrm{I}+\mathrm{Br}^{*}$ or $\mathrm{I}+\mathrm{Br}$, is accomplished by the split-operator method. ${ }^{42}$

The time-integrated flux over $[-\infty, t]$ yields the dissociation probability

$$
P_{i}(t)=\int_{-\infty}^{t} J_{i}\left(t^{\prime}\right) d t^{\prime},
$$

where the probability flux is obtained from

$$
J_{i}(t)=\frac{\hbar}{m} \operatorname{Im}\left[\psi_{i}^{*}(x, t) \frac{\partial \psi_{i}(x, t)}{\partial x}\right]_{x=x_{d}},
$$

where $m$ is the reduced mass of IBr. In the following, we choose $x_{d}=14$ a.u. and evaluate $P_{i}(t=7.5 \mathrm{ps})$. Although the wave packet, essentially, has passed the crossing region in less than $100 \mathrm{fs}$, the long propagation time is required due to small long-lived components of the wave packet. The intensity of each pulse in the two-pulse pump sequence is $1.7 \times 10^{10} \mathrm{~W} / \mathrm{cm}^{2}$ (with a constant transition dipole moment of 1 a.u.), such that the calculations are performed in the weak-field regime.

\section{RESULTS AND DISCUSSION}

Figure 2 shows the relative yield of $\mathrm{Br}^{*}$ as a function of time delay between two 2 fs Gaussian pulses. The relative normalized yield is defined as: $P\left(\mathrm{Br}^{*}\right) /\left(P\left(\mathrm{Br}^{*}\right)+P(\mathrm{Br})\right)$ where $P\left(\mathrm{Br}^{*}\right)$ and $P(\mathrm{Br})$ are the total dissociation probability in the

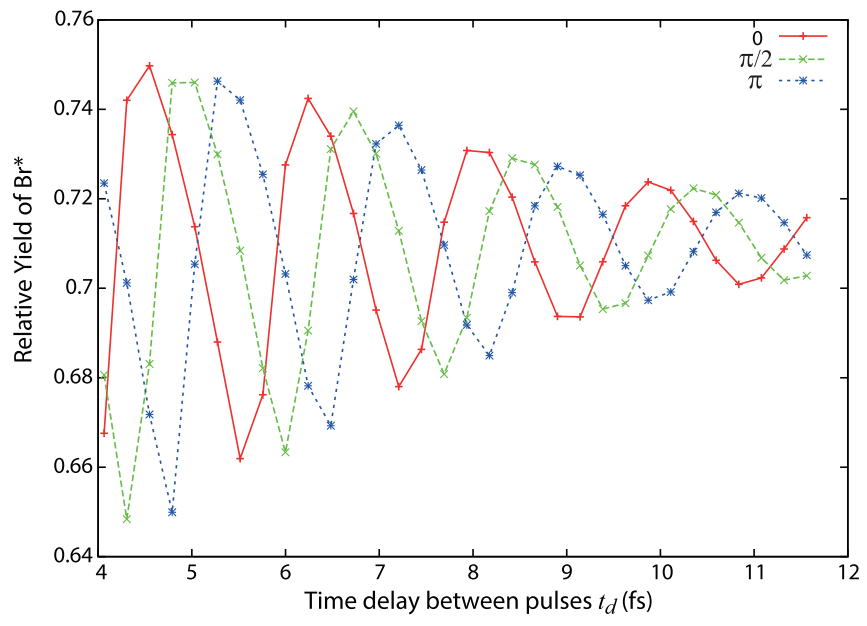

FIG. 2. Relative normalized yield of $\mathrm{Br}^{*}$ as a function of time delay between the two pulses in a two-pulse sequence. The yield is shown for three different values of the relative phase $\phi_{r}: 0, \pi / 2$, and $\pi$. two channels $\mathrm{I}+\mathrm{Br}^{*}$ and $\mathrm{I}+\mathrm{Br}$, respectively. The results are also shown for different relative phases $\phi_{r}$. It is observed that the branching ratio oscillates with a period of about $1.7 \mathrm{fs}$. The total population (not shown) in the excited states oscillates in exactly the same way as a function of time delay between the pulses. ${ }^{36}$

Each pulse in the two-pulse sequence creates a wave packet and the interference between the two excited wave packets $G_{1}$ and $G_{2}$ gives rise to a time-dependent overlap term in the total population, given by $2 \operatorname{Re}\left\{\left\langle G_{1}(t) \mid G_{2}(t)\right\rangle\right\}$. For nonoverlapping Gaussian pulses with a temporal duration of $10 \mathrm{fs}$ or longer, the branching ratio is basically identical to that of a single pulse, because significant interference (overlap) between the excited wave packets is lost for longer time delays.

The fast oscillations in Fig. 2 are associated with the interference term between the wave packets. The decay of the overlap in position-momentum space is modulated by a factor which oscillates at high frequency, related to the relative phase of the wave packets. Assume instantaneous, $\delta$-pulse, excitation then the wave packets are to an excellent approximation Gaussian wave packets. The phase associated with the time-evolution of a Gaussian wave packet $G$ is given by the classical action. In the short-time limit, the relative phase is given by $\exp \left(-i V t_{d} / \hbar\right)^{14}$ where $V$ is the value of the $B$-state potential at the Franck-Condon point. This relative phase oscillates with a period a little below $2 \mathrm{fs}$, in good agreement with the fast oscillations in Fig. 2.

We now consider the basic properties of the two-pulse sequence that determines the branching ratio. To that end, it is important to notice that the frequency distribution of the laser field is modified when the time delay, $t_{d}$, or the relative phase, $\phi_{r}$, in Eq. (4) is changed. Fourier transforming the electric field, we obtain the modulus and phase of the frequency distribution, $A(\omega) e^{i \phi(\omega)}$. From the modulus $A(\omega)=\left|A(\omega) e^{i \phi(\omega)}\right|$ (equivalent to a laser phase of zero) we have generated the electric fields according to Eq. (1),

$$
\mathcal{E}(t)=\mathcal{E}_{0} \operatorname{Re}\left[\int_{-\infty}^{\infty} A(\omega) e^{-i \omega t} d \omega\right] .
$$

Figure 3 shows, at two different time delays, electric fields which clearly differ from the two-pulse sequences. However, these fields give (within numerical accuracy) the same branching ratios as the two-pulse pump sequences at time delays of 4.55 fs and $5.51 \mathrm{fs}$, respectively. Thus, the obtained branching ratio is completely determined by the modulus of the frequency distributions and independent of the detailed pulse shape. The changing branching ratio in Fig. 2 is accordingly a direct reflection of a frequency distribution which changes when the time delay or the relative phase are changed.

To that end, it is also relevant to mention the frequency resolved (cw) results from the literature. Thus, at $530 \mathrm{~nm}$ the branching is 0.67 , and the branching ratio increases with decreasing wavelength to 0.76 at $470 \mathrm{~nm} .{ }^{41}$ The branching ratio obtained with the two-pulse sequence is identical to an average over the cw results, weighted by $|A(\omega)|^{2}$. This is equivalent to the result contained in Eq. (2). This result was, however, not so evident since previous formal proofs ${ }^{12,13}$ explicitly only considered direct fragmentation, i.e., all 

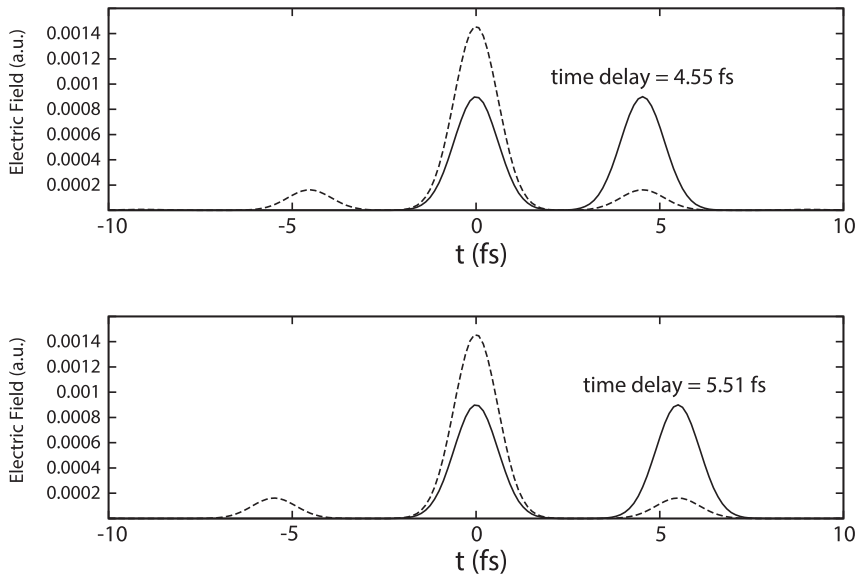

FIG. 3. Electric fields (dashed lines) with same frequency distribution as the two-pulse sequence at time delays of $4.55 \mathrm{fs}$ and $5.51 \mathrm{fs}$ (solid lines), respectively. These fields give the same branching ratios as the two-pulse sequences.

arguments were based on standard stationary scattering states associated with a single potential energy surface. In the present work we considered a more complicated case of two coupled excited-state continua.

Finally, it is interesting to consider, in more detail, how we can explain the change in branching ratio shown in Fig. 2. The Landau-Zener formalism offers insight into the crossing probability within a semiclassical framework based on a single classical trajectory. ${ }^{40,43}$ However, the applicability of this formalism goes beyond the trajectory assumption; when this assumption is abandoned and replaced by quantum dynamics described by a Gaussian wave packet, a remarkable agreement with the simple Landau-Zener formula is observed ${ }^{44}$ provided the speed of the classical particle is replaced by the quantum mechanical expectation value of the speed,

$$
P_{\mathrm{LZ}}=\exp \left(-2 \pi\left|V_{12}\right|^{2} / \hbar\left|\beta_{2}-\beta_{1}\right| v_{c}\right),
$$

where $V_{12}$ is the (constant) coupling potential between the diabatic states, $\beta_{1}$ and $\beta_{2}$ are the derivatives of the diabatic states at the crossing, and $v_{c}$ is the expectation value of the speed at the time of the crossing. In practice, we evaluate this quantity as follows: We determine the time when the average internuclear position is equal to 6 a.u., for this time we calculate the expectation value of the momentum $\langle p\rangle_{c}$ and $v_{c}=\langle p\rangle_{c} / m$, where $m$ is the reduced mass.

From Table I, it is observed that the Landau-Zener formula, Eq. (9) quite nicely reproduces the exact crossing probabilities. The error is less than a few percent and the largest error of about $6 \%$ at $t_{d}=5.51 \mathrm{fs}$ is obtained when the mo-

TABLE I. Comparison of crossing probabilities obtained numerically exact or from the Landau-Zener formula, for wave packets created by a two-pulse sequence of 2 fs Gaussians at different time delays.

\begin{tabular}{lcc}
\hline \hline Time delay (fs) & $P$ (exact) & $P_{\mathrm{LZ}}$ \\
\hline 4.55 & 0.750 & 0.754 \\
5.51 & 0.662 & 0.705 \\
100.0 & 0.715 & 0.735 \\
\hline \hline
\end{tabular}
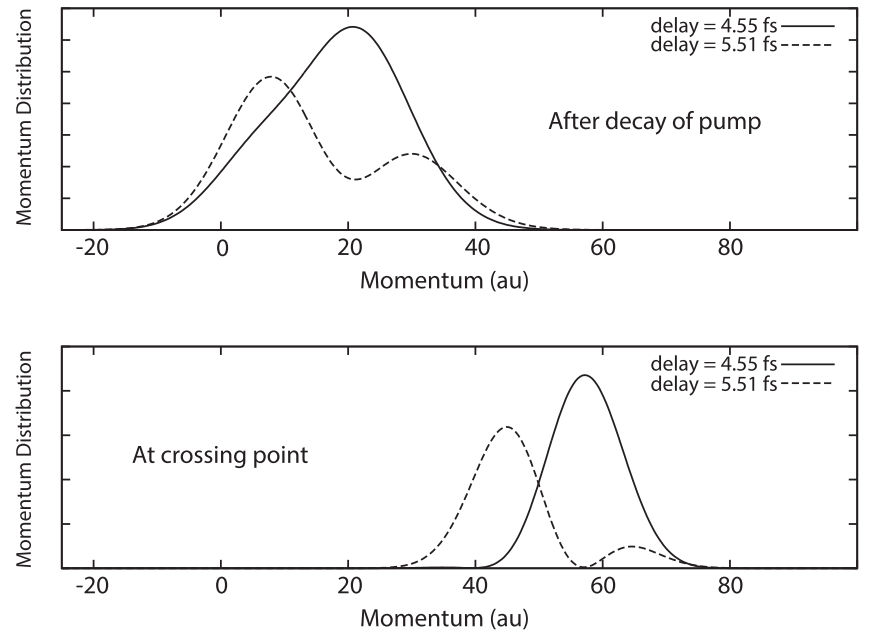

FIG. 4. Momentum distributions of the wave packets right after the excitation with a two-pulse pump sequence at the time delays of $4.55 \mathrm{fs}$ and $5.51 \mathrm{fs}$, respectively, and at the time when the average position is at the crossing.

mentum distribution (see Figure 4) shows the largest deviation from a symmetrical distribution where the average coincides with the maximum in the distribution. Thus, the dynamics of the crossing is to a good approximation determined by the average momentum at the crossing. The result at a time delay of $100 \mathrm{fs}$ is identical to the crossing probability obtained after excitation with a single 2 fs Gaussian pulse.

\section{CONCLUSIONS}

While the pulse energy is kept constant, we have demonstrated that a photofragmentation branching ratio can be controlled by the time delay in a pulse train. This should be contrasted with the traditional $\mathrm{cw}$ photochemistry where different photon energies are required in order to change a branching ratio.

We considered non-overlapping pulses, thus the energy of the pulse train is constant for all time delays. The frequency distribution does, however, depend on the time delay. Thus, different time delays translate into different frequency distributions, however, all with the same pulse energy. The photofragmentation dynamics of $\mathrm{IBr}$, involving two coupled excited-state continua, was considered. For this process, induced by a weak field and starting from the vibrational ground state, we showed that the change in the branching ratio (between two electronic states) as a function of time delay is only a reflection of a changing frequency distribution of the pulse train. Thus, the branching ratio does not depend on the detailed pulse shape. This supports the conclusions of previous work, ${ }^{12,13}$ however, here extended to a more complicated situation with coupled excited-state continua.

A two-pulse sequence with a short delay creates interference among the excited wave packets. These interferences lead to different "initial" momentum distributions, that is, a branching ratio which depends on time delay. A fast time dependence in the branching ratio as a function of time delay was observed and it follows the time dependence in the relative phase of the excited wave packets, which is associated with the interference term between the wave packets. 
The control scheme presented in this paper shares some relation to earlier interferometric experiments ${ }^{33,34}$ where it was demonstrated that wave packet interference could change the population in a bound vibrational state.

Finally, we showed that a simple extension of LandauZener theory reproduces nicely the branching ratio when the average momentum at the crossing gives a good description of the momentum distribution.

${ }^{1}$ S. A. Rice and M. Zhao, Optical Control of Molecular Dynamics (Wiley, New York, 2000).

${ }^{2}$ M. Shapiro and P. Brumer, Quantum Control of Molecular Processes (Wiley, New York, 2012).

${ }^{3}$ C. Brif, R. Chakrabarti, and H. Rabitz, New J. Phys. 12, 075008 (2010).

${ }^{4}$ A. Assion, T. Baumert, M. Bergt, T. Brixner, B. Kiefer, V. Seyfried, M. Strehle, and G. Gerber, Science 282, 919 (1998).

${ }^{5}$ T. C. Weinacht, J. Ahn, and P. H. Bucksbaum, Nature 397, 233 (1999).

${ }^{6}$ R. J. Levis, G. M. Mekir, and H. Rabitz, Science 292, 709 (2001).

${ }^{7}$ G. Vogt, G. Krampert, P. Niklaus, P. Nuernberger, and G. Gerber, Phys. Rev. Lett. 94, 068305 (2005).

${ }^{8}$ I. Barth and J. Manz, Angew. Chem., Int. Ed. 45, 2962 (2006).

${ }^{9}$ A. S. Alnaser et al., Nat. Commun. 5, 3800 (2014).

${ }^{10}$ B. J. Sussman, D. Townsend, M. Yu. Ivanov, and A. Stolow, Science 314, 278 (2006).

${ }^{11}$ M. E. Corrales, J. González-Vázquez, G. Balerdi, I. R. Sola, R. de Nalda, and L. Bañares, Nat. Chem. 6, 785 (2014).

${ }^{12}$ P. Brumer and M. Shapiro, Chem. Phys. 139, 221 (1989).

${ }^{13}$ M. Shapiro and P. Brumer, J. Phys. Chem. A 105, 2897 (2001).

${ }^{14}$ N. E. Henriksen, Adv. Chem. Phys. 91, 433 (1995).

${ }^{15}$ P. Brumer and M. Shapiro, Chem. Phys. Lett. 126, 541 (1986).

${ }^{16}$ B. Amstrup and N. E. Henriksen, J. Chem. Phys. 97, 8285 (1992).

${ }^{17}$ N. E. Henriksen and B. Amstrup, Chem. Phys. Lett. 213, 65 (1993).

${ }^{18}$ M. Shapiro and P. Brumer, J. Chem. Phys. 98, 201 (1993).

${ }^{19}$ B. Amstrup and N. E. Henriksen, J. Chem. Phys. 105, 9115 (1996).

${ }^{20}$ D. J. Tannor and S. A. Rice, J. Chem. Phys. 83, 5013 (1985).
${ }^{21}$ A. K. Tiwari, K. B. Møller, and N. E. Henriksen, Phys. Rev. A 78, 065402 (2008).

${ }^{22}$ C. C. Shu, T. Rozgonyi, L. González, and N. E. Henriksen, J. Chem. Phys. 136, 174303 (2012).

${ }^{23}$ V. I. Prokhorenko, A. M. Nagy, S. A. Waschuk, L. S. Brown, R. R. Birge, and R. J. Dwayne Miller, Science 313, 1257 (2006).

${ }^{24}$ V. I. Prokhorenko, A. M. Nagy, L. S. Brown, and R. J. Dwayne Miller, Chem. Phys. 341, 296 (2007).

${ }^{25}$ G. Katz, M. A. Ratner, and R. Kosloff, New J. Phys. 12, 015003 (2010).

${ }^{26}$ C. A. Arango and P. Brumer, J. Chem. Phys. 138, 071104 (2013).

${ }^{27}$ M. Spanner, C. A. Arango, and P. Brumer, J. Chem. Phys. 133, 151101 (2010).

${ }^{28}$ C. C. Shu and N. E. Henriksen, J. Chem. Phys. 134, 164308 (2011).

${ }^{29}$ C. C. Shu and N. E. Henriksen, J. Chem. Phys. 136, 044303 (2012),

${ }^{30}$ A. K. Tiwari, D. Dey, and N. E. Henriksen, Phys. Rev. A 89, 023417 (2014).

${ }^{31}$ I. Levy, M. Shapiro, and P. Brumer, J. Chem. Phys. 93, 2493 (1990).

${ }^{32}$ E. J. Heller, Acc. Chem. Res. 14, 368 (1981).

${ }^{33}$ N. F. Scherer, R. J. Carlson, A. Matro, M. Du, A. J. Ruggiero, V. R. Rochin, J. A. Cina, G. R. Fleming, and S. A. Rice, J. Chem. Phys. 95, 1487 (1991).

${ }^{34}$ K. Ohmori, Y. Sato, E. E. Nikitin, and S. A. Rice, Phys. Rev. Lett. 91, 243003 (2003)

${ }^{35}$ E. Skovsen, M. Machholm, T. Ejdrup, J. Thøgersen, and H. Stapelfeldt, Phys. Rev. Lett. 89, 133004 (2002).

${ }^{36}$ H. Metiu and V. Engel, J. Opt. Soc. Am. B 7, 1709 (1990).

${ }^{37}$ T. Buckup, J. Hauer, J. Voll, R. Vivie-Riedle, and M. Motzkus, Faraday Discuss. 153, 213 (2011).

${ }^{38}$ T. Grinev and P. Brumer, J. Chem. Phys. 140, 124307 (2014).

${ }^{39}$ C. Sanz-Sanz, G. W. Richings, and G. A. Worth, Faraday Discuss. 153, 275 (2011).

${ }^{40}$ E. Wrede, S. Laubach, S. Schulenburg, A. Brown, E. R. Wouters, A. J. Orr-Ewing, and M. N. R. Ashfold, J. Chem. Phys. 114, 2629 (2001).

${ }^{41}$ H. Guo, J. Chem. Phys. 99, 1685 (1993).

${ }^{42}$ R. Kosloff, J. Phys. Chem. 92, 2087 (1988).

${ }^{43}$ N. E. Henriksen and F. Y. Hansen, Theories of Molecular Reaction Dynamics (Oxford University Press, Oxford, 2008).

${ }^{44}$ N. E. Henriksen, Chem. Phys. Lett. 197, 620 (1992). 